\title{
In vitro and in vivo antitumor effects of chloroquine on oral squamous cell carcinoma
}

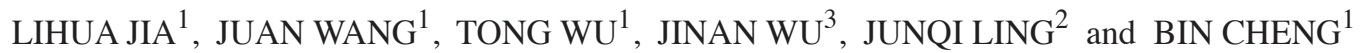 \\ Departments of ${ }^{1}$ Oral Medicine and ${ }^{2}$ Endodontics, Guanghua School of Stomatology, Guangdong Provincial Key Laboratory \\ of Stomatology, Sun Yat-Sen University, Guangzhou, Guangdong 510060; ${ }^{3}$ Department of Oral and Maxillofacial \\ Surgery, Affiliated Zhongshan Hospital, Sun Yat-Sen University, Zhongshan, Guangdong 528403, P.R. China
}

Received April 28, 2016; Accepted April 19, 2017

DOI: $10.3892 / \mathrm{mmr} .2017 .7342$

\begin{abstract}
Chloroquine, which is a widely used antimalarial drug, has been reported to exert anticancer activity in some tumor types; however, its potential effects on oral squamous cell carcinoma (OSCC) remain unclear. The present study aimed to explore the effects and possible underlying mechanisms of chloroquine against OSCC. MTT and clonogenic assays were conducted to evaluate the effects of chloroquine on the human OSCC cell lines SCC25 and CAL27. Cell cycle progression and apoptosis were detected using flow cytometry. Autophagy was monitored using microtubule-associated protein 1A/1B-light chain 3 as an autophagosomal marker. In order to determine the in vivo antitumor effects of chloroquine on OSCC, a CAL27 xenograft model was used. The results demonstrated that chloroquine markedly inhibited the proliferation and the colony-forming ability of both OSCC cell lines in a dose- and time-dependent manner in vitro. Chloroquine also disrupted the cell cycle, resulting in the cell cycle arrest of CAL27 and SCC 25 cells at $G_{0} / G_{1}$ phase, via downregulation of cyclin D1. In addition, chloroquine inhibited autophagy, and induced autophagosome and autolysosome accumulation in the cytoplasm, thus interfering with degradation; however, OSCC apoptosis was barely affected by chloroquine. The results of the in vivo study demonstrated that chloroquine effectively inhibited OSCC tumor growth in the CAL27 xenograft model. In conclusion, the present study reported the in vitro and
\end{abstract}

Correspondence to: Professor Bin Cheng, Department of Oral Medicine, Guanghua School of Stomatology, Guangdong Provincial Key Laboratory of Stomatology, Sun Yat-Sen University, 56 Lingyuanxi Road, Guangzhou, Guangdong 510060, P.R. China E-mail: chengbin@mail.sysu.edu.cn

Professor Junqi Ling, Department of Endodontics, Guanghua School of Stomatology, Guangdong Provincial Key Laboratory of Stomatology, Sun Yat-Sen University, 56 Lingyuanxi Road, Guangzhou, Guangdong 510060, P.R. China

E-mail: lingjunqi@mail.sysu.edu.cn

Key words: chloroquine, oral squamous cell carcinoma, cell cycle, cyclin D1, autophagy, LC3B, CAL27 xenograft model in vivo antitumor effects of chloroquine on OSCC, and the results indicated that chloroquine may be considered a potent therapeutic agent against human OSCC.

\section{Introduction}

Chloroquine has been widely used as a potent antimalarial and amebicidal drug since 1934, and has been proven to be well tolerated in humans. Previous studies have suggested that chloroquine may be regarded as an effective anticancer drug, according to the 'old drugs-new uses' repositioning strategy, since chloroquine has been reported to exert numerous biological effects, including inhibiting cell growth and/or inducing cell death of various types of cancer cells, overcoming chemoresistance, increasing radiosensitivity and eliminating cancer stem cells $(1,2)$. The role of autophagy has previously been demonstrated in cancer development and therapeutic resistance; it serves a role as a protective cell mechanism by eliminating excessive or unnecessary proteins and injured or aged organelles in the microenviroment of tumor progression, as induced by nutrient deprivation, hypoxia or therapeutic stress. Chloroquine has been reported to act as an effective agent that inhibits autophagy by interfering with the degradative functions of lysosomes, resulting in the accumulation of damaged autolysosomes. The inhibition of autophagy by chloroquine may lead to accelerated tumor cell death, reduced chemoresistance and increased radiosensitivity (3).

Oral squamous cell carcinoma (OSCC) is the sixth most common malignancy worldwide, and accounts for $>90 \%$ of cancers in the oral cavity (4). Surgery, with or without adjuvant chemoradiotherapy, remains the gold standard for treatment of OSCC, which exerts curative effects, particularly during the early stages of OSCC. However, there are serious side effects associated with current therapeutic regimens, including no significant improvement of the 5-year survival rate, and a significantly increased risk of developing subsequent primary or recurrent tumors. Pignon et al conducted a meta-analysis of 63 trials (10,741 patients) and reported that chemotherapy, alongside locoregional treatment, provided an absolute survival benefit of $4 \%$ at 2 and 5 years (5); however, the efficacy of neoadjuvant chemotherapy, including cisplatin, 5-fluorouracil, paclitaxel and carboplatin, remains controversial (6-8). 
There remains an urgent demand for more effective agents to better combat OSCC. The antimalarial drug chloroquine, alone or in combination with other agents, has been reported to exert antitumor efficacy on several types of cancer, including breast, colon and liver cancer (9-14). Therefore, the present study aimed to explore the effects of chloroquine on OSCC, and the potential molecular targets and pathways associated with chloroquine treatment of OSCC.

\section{Materials and methods}

Materials. Chloroquine, which was purchased from Sigma-Aldrich; Merck KGaA (Darmstadt, Germany), was solubilized in phosphate-buffered saline (PBS; 0.1 M stock solution stored at $-20^{\circ} \mathrm{C}$ ) and was used within 2 weeks.

Culture conditions. Two human oral squamous cancer cell lines (SCC25, CAL27) were used in the present study. CAL27 (ATCC number: CRL-2095) and SCC25 (ATCC number: CRL-1628) cell lines were purchased from the American Type Culture Collection (Manassas, VA, USA). SCC25 cells were routinely grown in Dulbecco's modified Eagle's medium (DMEM)/F-12 (1:1; Gibco; Thermo Fisher Scientific, Inc., Waltham, MA, USA) supplemented with $10 \%$ fetal bovine serum (Gibco; Thermo Fisher Scientific, Inc.) and $2 \mathrm{mM}$ L-glutamine. CAL27 cells were cultured in DMEM (Gibco; Thermo Fisher Scientific, Inc.). Cells were maintained at $37^{\circ} \mathrm{C}$ in a humidified atmosphere containing $95 \%$ air and $5 \%$ $\mathrm{CO}_{2}$. SCC25 and CAL27 cells were seeded in cell plates and cultured for $24 \mathrm{~h}$, after which the medium was removed and replaced with DMEM/F-12 supplemented with $0.5 \%$ FBS for SCC25 cells, or with DMEM for CAL27 cells, with or without chloroquine. Untreated cells were considered the control group.

Cell proliferation assay. The effects of chloroquine on cell proliferation were evaluated using the MTT assay (BioSource International, Inc., Camarillo, CA, USA). Cells $\left(5 \times 10^{3}\right.$ cells/well) were plated into 96 -well plates and treated with various doses of chloroquine, or culture medium without chloroquine as a vehicle control, for 24,48 and $72 \mathrm{~h}$ at $37^{\circ} \mathrm{C}$. Subsequently, an MTT assay was conducted according to the manufacturer's protocol and absorbance [optical density (OD)] was measured at $490 \mathrm{~nm}$ using a microplate spectrophotometer (Thermo Fisher Scientific, Inc.). The inhibitory effects of chloroquine on OSCC cell proliferation were calculated using the following formula: Inhibitory rate $=(1$-chloroquine-treated $\mathrm{OD}_{490} /$ control $\left.\mathrm{OD}_{490}\right) \times 100 \%$.

Clonogenic assay. Clonogenic assays were performed as described previously (15). Briefly, cells were seeded in triplicate into 6-well plates at a density of 1,000 cells/well. The cells were then treated with 10 and $30 \mu \mathrm{M}$ chloroquine, or vehicle control, for 1 week at $37^{\circ} \mathrm{C}$. Subsequently, the cell colonies were stained with a solution containing $0.5 \%$ crystal violet and $25 \%$ methanol, and washed three times with tap water. Colonies consisting of $>50$ cells were counted under a light microscope.

Cell cycle analysis. Flow cytometric analyses were performed as described previously (15), in order to determine the cell cycle distribution of chloroquine-treated and untreated cells. A total of $2 \times 10^{5}$ OSCC cells were seeded in 6-well plates and cultured for $24 \mathrm{~h}$ at $37^{\circ} \mathrm{C}$, treated with or without 10 and $30 \mu \mathrm{M}$ chloroquine for $24 \mathrm{~h}$, and harvested. Cells were fixed and stained for total DNA content with a solution containing $50 \mu \mathrm{g} / \mathrm{ml}$ propidium iodide (PI) and $100 \mu \mathrm{g} / \mathrm{ml}$ RNase I (BD Biosciences, San Diego, CA, USA) in PBS for $30 \mathrm{~min}$ at $37^{\circ} \mathrm{C}$. Cell cycle distribution was immediately analyzed using a Cytomics FC500 MCL with CXP software 1.0 (Beckman Coulter, Inc., Brea, CA, USA). The experiment was performed three times, and the ratio of cells in $\mathrm{G}_{0} / \mathrm{G}_{1}$, intra-S and $\mathrm{G}_{2} / \mathrm{M}$ phases was expressed as the mean \pm standard deviation.

Apoptosis analysis. OSCC cells $\left(2 \times 10^{5}\right)$ cultured in 6-well plates were treated with or without 10 and $30 \mu \mathrm{M}$ chloroquine for $24 \mathrm{~h}$ at $37^{\circ} \mathrm{C}$. Harvested cells were stained with fluorescein isothiocyanate (FITC)-conjugated Annexin V and PI for 5 min at room temperature, according to the manufacturer's protocol (Annexin V FITC Apoptosis Detection kit; BD Pharmingen; BD Biosciences). The population of AnnexinV $\mathrm{PI}^{-}$viable cells and Annexin $\mathrm{V}^{+}$apoptotic cells was evaluated by flow cytometry; the latter were considered apoptotic cells. Data were immediately collected using a Cytomics FC500 MCL with CXP software 1.0 (Beckman Coulter, Inc.). The experiment was performed three times, and the ratio of apoptotic cells was expressed as the mean \pm standard deviation.

Reverse transcription-quantitative polymerase chain reaction $(R T-q P C R)$. To determine the mRNA expression levels of microtubule-associated proteins 1A/1B light chain 3B (LC3B) and cyclin D1 in the cells RT-qPCR was conducted. OSCC cells treated with or without 10 and $30 \mu \mathrm{M}$ chloroquine for $24 \mathrm{~h}$ at $37^{\circ} \mathrm{C}$ were harvested, and total RNA from each sample was isolated with TRIzol (Invitrogen; Thermo Fisher Scientific, Inc.) according to the manufacturer's protocol. The concentration and quality of RNA was measured using a spectrophotometer at 260 and $280 \mathrm{~nm}$. Briefly, $2 \mu \mathrm{g}$ total RNA from each sample underwent RT (reaction volume, $20 \mathrm{ml}$ ), using an RT system (Roche Diagnostics, Mannheim, Germany) according to the manufacturer's protocol. SYBR Green Assay kit (Roche Diagnostics) was used to conduct qPCR, according to the manufacturer's protocol. The PCR primer sequences were designed by Takara Biotechnology Co., Ltd. (Dalian, China), as follows: GAPDH, forward AGGCTAGCTGGCCCGATTTC, reverse TGGCAACAA TATCCACTTTACCAGA; LC3B, forward AAACGCATT TGCCATCACA, reverse GGACCTTCAGCAGTTTAC AGTCAG; and cyclin D1, forward GACTCTCATTCGGGA TGATTGGA and reverse TTTGGTTCGGCAGCTTGCTA. qPCR was conducted using the LightCycler480 instrument (Roche Diagnostics) and the results were analyzed using LightCycler480 software 1.5 (Roche Diagnostics). The thermal cycling conditions used during PCR amplification were as follows: $5 \mathrm{~min}$ at $95^{\circ} \mathrm{C}$, followed by $40-45$ cycles of denaturation at $95^{\circ} \mathrm{C}$ for $10 \mathrm{sec}$, annealing at $60^{\circ} \mathrm{C}$ for $20 \mathrm{sec}$ and extension at $72^{\circ} \mathrm{C}$ for $20 \mathrm{sec}$, followed by $95^{\circ} \mathrm{C}$ for $5 \mathrm{sec}$ and $65^{\circ} \mathrm{C}$ for $1 \mathrm{~min}$; the temperature was then increased from 65 to $95^{\circ} \mathrm{C}$ for the melt curve analysis and was then decreased to $40^{\circ} \mathrm{C}$ for $10 \mathrm{sec}$ to cool the samples. Cyclin D1 and LC3 expression were analyzed using the $2^{-\Delta \Delta \mathrm{Cq}}$ method, and 
expression levels were normalized to GAPDH expression in each sample (16).

Immunofluorescence. OSCC cells seeded into 24-well plates were treated with or without 10 and $30 \mu \mathrm{M}$ chloroquine for $24 \mathrm{~h}$ at $37^{\circ} \mathrm{C}$, after which the cells were washed with PBS and fixed with methanol at $-20^{\circ} \mathrm{C}$ for $20 \mathrm{~min}$. The cells were then permeabilized with $0.1 \%$ Tween- 20 for $15 \mathrm{~min}$ at room temperature and were blocked with $0.2 \%$ bovine serum albumin (Gibco; Thermo Fisher Scientific, Inc.) for $1 \mathrm{~h}$ at room temperature. The cells were incubated with anti-LC3B antibody (2775S; 1:200 dilution; Cell Signaling Technology, Inc., Beverly, MA, USA) at $4^{\circ} \mathrm{C}$ overnight, were washed with PBS and were then incubated with Alexa Fluor 647-conjugated goat anti-rabbit antibody (ab150079; 1:1,000 dilution; Abcam, Cambridge, UK) for $1 \mathrm{~h}$ at room temperature. DAPI (10236276001; $300 \mathrm{nM}$; Roche Diagnostics) was used to counterstain nuclei for $1 \mathrm{~min}$ at room temperature. Cells were examined under a fluorescence microscope (Leica DMI4000B; Leica Microsystems GmbH, Wetzlar, Germany).

Western blot analysis. Cells were treated with chloroquine $(0,10$ and $30 \mu \mathrm{M})$ in 6 -well plates for $24 \mathrm{~h}$ at $37^{\circ} \mathrm{C}$ and were harvested by scraping the culture dishes with a cell scraper. Subsequently, cells were lysed with $150 \mu 1$ radioimmunoprecipitation assay buffer containing various protease inhibitors for $30 \mathrm{~min}$ on ice (Pierce; Thermo Fisher Scientific, Inc.). Following centrifugation at $12,000 \mathrm{x}$ g for $10 \mathrm{~min}$ at $4^{\circ} \mathrm{C}$, the clear supernatant was collected and used as the cell protein extract. Protein concentration was determined using the bicinchoninic acid protein assay kit (Nanjing Keygen Biotech Co., Ltd., Nanjing, China). Protein $(40 \mu \mathrm{g})$ from control cells and chloroquine-treated cells were separated by $12 \%(\mathrm{w} / \mathrm{v})$ SDS-PAGE and were electroblotted onto polyvinylidene fluoride membranes (Bio-Rad Laboratories, Inc., Hercules, CA, USA). For immunodetection, membranes were blocked with $5 \%$ non-fat milk in TBS containing $0.1 \%$ Tween-20 for $1 \mathrm{~h}$ at room temperature prior to incubation with rabbit monoclonal anti-LC3B (2775S) and anti-GAPDH (2118) antibodies (1:1,000 dilution; Cell Signaling Technology, Inc.) at $4^{\circ} \mathrm{C}$ overnight, followed by incubation with goat anti-rabbit horseradish peroxidase (HRP)-conjugated secondary antibody (sc-2004; 1:5,000 dilution; Santa Cruz Biotechnology, Inc., Dallas, TX, USA) for $1 \mathrm{~h}$ at room temperature. Antibody binding was determined using an enhanced chemiluminescence substrate to HRP (Invitrogen; Thermo Fisher Scientific, Inc.). The anti-LC3B (2775S) antibody produced two clear bands representing LC3-I (16 kDa) and LC3-II (14 kDa). The expression levels of LC3-II are expressed as the density measured by ImageJ 1.46 software (National Institutes of Health, Bethesda, MD, USA), standardized to the density of GAPDH.

Xenograft murine model analysis. The present study was approved by the ethics committee of Guanghua School of Stomatology, Sun Yat-Sen University (Guangzhou, China). A total of 10 female and 10 male BALB/c nude mice (age, 4 weeks; weight, 14-16 g) were purchased from the Guangdong Medical Laboratory Animal Center (Foshan, China), and were bred in the Animal Care Unit of Sun Yet-sun University under standard pathogen-free conditions. Mice were maintained under the following conditions: Temperature, $20-23^{\circ} \mathrm{C}$; relative humidity, 50-65\%; 12-h light/dark cycle; free access to food and water. Each mouse was subcutaneously inoculated with $5 \times 10^{6}$ CAL27 cells into the back next to the right front limb. After 10 days, the xenografts were identifiable as a mass $>6 \mathrm{~mm}$ in maximal diameter in all recipients.

CAL27-bearing BABL/c nude mice were randomly assigned into two groups ( $\mathrm{n}=10 /$ group). The treated mice were intraperitoneally injected with $50 \mathrm{mg} / \mathrm{kg}$ chloroquine daily, whereas the control mice were injected with normal saline. Tumor size was determined by caliper measurement of the largest and perpendicular diameters every 2 days, and tumor volumes were calculated according to the following formula: Volume $=\mathrm{axb}^{2} / 2$, where a refers to the largest superficial diameter and $\mathrm{b}$ refers to the smallest superficial diameter. The mice were sacrificed 24 days after treatment, and the tumors were removed and weighed.

Statistical analysis. The data are presented as the mean \pm standard deviation for at least three repeated individual experiments for each group. Statistical analysis of the in vitro results was determined by one-way analysis of variance followed by the least significant difference test, whereas the in vivo experiments were analyzed using a two-tailed Student's t test. Statistical analyses were conducted using SPSS 16.0 (SPSS, Inc., Chicago, IL, USA). $\mathrm{P}<0.05$ was considered to indicate a statistically significant difference.

\section{Results}

Chloroquine inhibits $O S C C$ cell proliferation and reduces colony formation in vitro. To determine the effects of chloroquine on human OSCC cell growth in vitro, MTT and clonogenic assays were performed using two OSCC cell lines. The results demonstrated that treatment with chloroquine (10, 20, 30 and $60 \mu \mathrm{M})$ significantly inhibited cell proliferation in a dose- and time-dependent manner in both OSCC cell lines $(\mathrm{P}<0.01$; Fig. $1 \mathrm{~A}$ and $\mathrm{B})$. After treatment with $60 \mu \mathrm{M}$ chloroquine for $72 \mathrm{~h}, \mathrm{SCC} 25$ and CAL27 cell growth was inhibited by 59.18 and $77.28 \%$, respectively, compared with the untreated controls. The half maximal inhibitory concentration $\left(\mathrm{IC}_{50}\right)$ values of chloroquine, following $48 \mathrm{~h}$ treatment, were 29.95 and 17.27 $\mu \mathrm{M}$ in SCC25 and CAL27 cells, respectively. Therefore, 10 and $30 \mu \mathrm{M}$ chloroquine, which encompassed concentrations above and below the $\mathrm{IC}_{50}$ values, were used in further experiments. Subsequently, the present study determined the colony-forming ability of SCC25 and CAL27 cells on 6-well cell culture plates in the presence or absence of chloroquine for a period of 1 week. Results indicated a marked decrease in OSCC cell colony formation following treatment with chloroquine, in a dose-dependent manner (Fig. 1C). Chloroquine $(10 \mu \mathrm{M})$ reduced the colony formation of SCC25 and CAL27 cells by 34.9 and $38.2 \%$, respectively $(\mathrm{P}<0.05)$ (data not shown). In addition, chloroquine $(30 \mu \mathrm{M})$ significantly reduced the colony formation of SCC25 and CAL27 cells by 98.1 and 99.4\%, respectively $(\mathrm{P}<0.01)$ (data not shown).

Chloroquine induces cell cycle arrest but not apoptosis. To further study the biological mechanisms underlying 
A
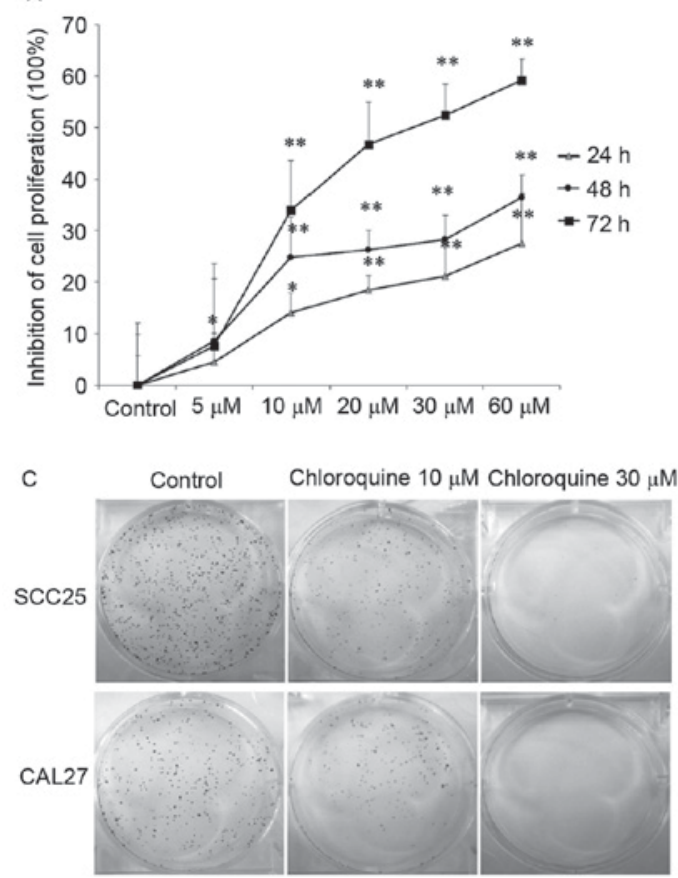

B

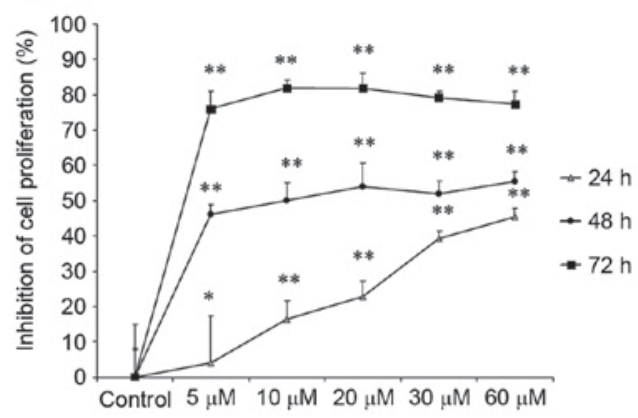

Figure 1. Chloroquine inhibits the proliferation and colony-forming ability of OSCC cells. (A) SCC25 and (B) CAL27 OSCC cells were treated with chloroquine $(0-60 \mu \mathrm{M})$ for 24,48 and $72 \mathrm{~h}(\mathrm{n}=10)$. Proliferation was detected using MTT assay, and inhibition of the rate of proliferation was calculated as the ratio to control untreated cells. The proliferative capacity of both OSCC cell lines was decreased by chloroquine in a dose- and time-dependent manner. "P $<0.05$, ${ }^{* *} \mathrm{P}<0.01$ vs. control cells. Results are presented as the mean \pm standard deviation of three independent experiments. (C) Clonogenic assay was performed on cells treated with or without 10 or $30 \mu \mathrm{M}$ chloroquine in 6-well plates $(\mathrm{n}=3)$. Chloroquine reduced colony formation at concentrations as low as $10 \mu \mathrm{M}$, and at the higher concentration of $30 \mu \mathrm{M}$, colony formation was reduced by $>90 \%$. OSCC, oral squamous cell carcinoma.

A

号

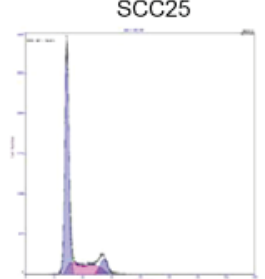

|

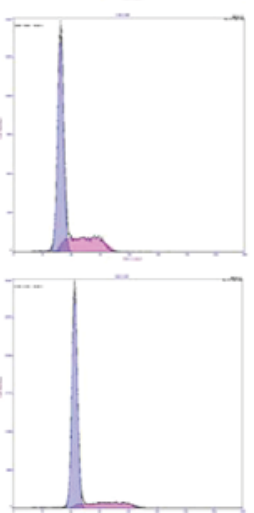

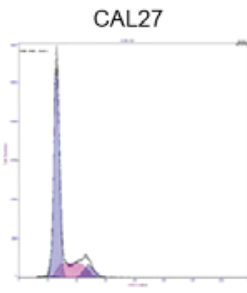
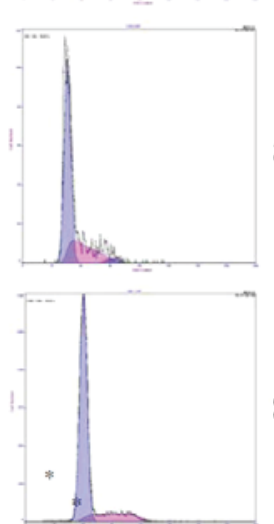

DNA contents $(\mathrm{PI})$
B

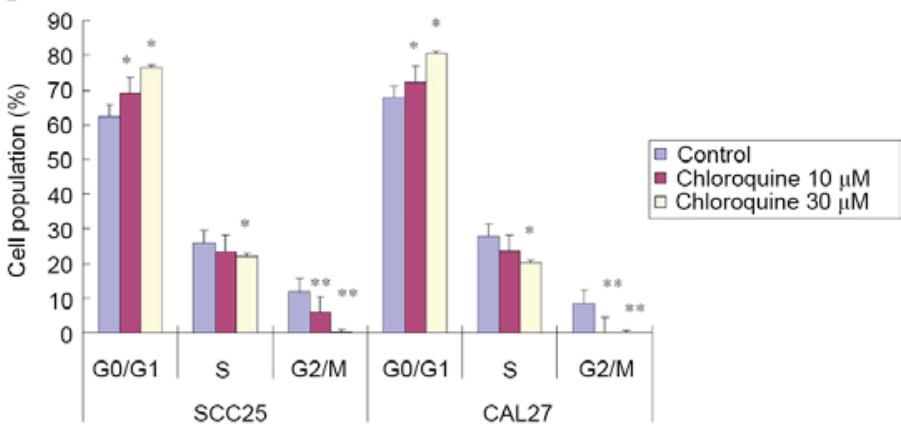

C

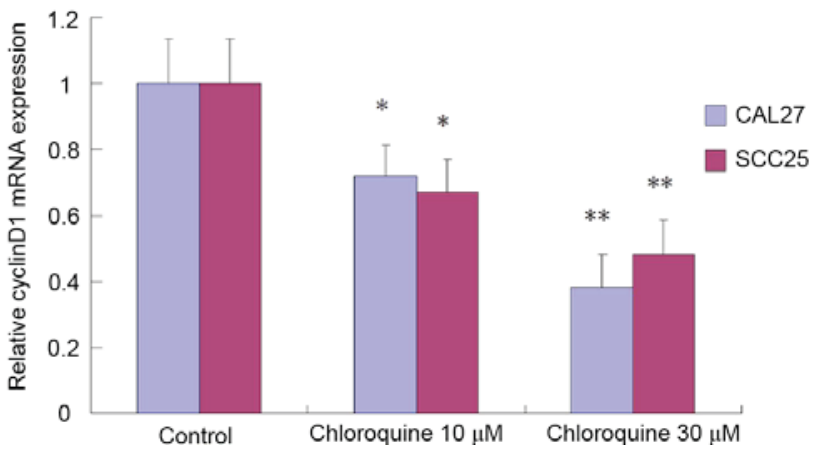

Figure 2. Chloroquine induces $\mathrm{G}_{0} / \mathrm{G}_{1}$ cell cycle arrest in OSCC cells. (A and B) SCC 25 and CAL27 OSCC cells were exposed to chloroquine ( 0 , 10 and $\left.30 \mu \mathrm{M}\right)$ for $24 \mathrm{~h}$ ( $\mathrm{n}=3$, three independent experiments). Cell cycle distribution was detected by PI staining using flow cytometry. Chloroquine treatment significantly decreased the percentage of cells in $\mathrm{G}_{2} / \mathrm{M}$ phase and increased the percentage of cells in $\mathrm{G}_{0} / \mathrm{G}_{1}$ phase in both OSCC cell lines compared with in the control cells. ${ }^{*} \mathrm{P}<0.05,{ }^{* *} \mathrm{P}<0.01$ vs. control cells. (C) mRNA expression levels of cyclin D1 were quantified by quantitative polymerase chain reaction in SCC25 and Cal27 cells $(\mathrm{n}=3)$. Cells treated with chloroquine exhibited a dose-dependent decrease in cyclin $\mathrm{D} 1$ expression. ${ }^{*} \mathrm{P}<0.05$, ${ }^{* *} \mathrm{P}<0.01 \mathrm{vs}$. control cells. OSCC, oral squamous cell carcinoma. 
chloroquine-induced growth inhibition, the present study evaluated the alterations in cell cycle progression and apoptosis by flow cytometric analysis (Figs. 2 and 3). Treatment with chloroquine significantly decreased the percentage of cells in $\mathrm{G}_{2} / \mathrm{M}$ phase and increased the percentage of cells in $\mathrm{G}_{0} / \mathrm{G}_{1}$ phase in both OSCC cell lines compared with control cells $(\mathrm{P}<0.05$; Fig. $2 \mathrm{~A}$ and $\mathrm{B})$. The chloroquine-associated $\mathrm{G}_{1}$ cell cycle arrest was dose-dependent. However, the chloroquine-induced growth inhibition was not associated with the induction of apoptosis. Apoptosis was detected in $<3 \%$ of OSCC cells treated with chloroquine; however, there was an insignificant increase in the number of apoptotic cells in the chloroquine groups compared with in untreated cells (Fig. 3).

The present study also determined whether chloroquine was able to induce alterations in molecular signaling associated with the $\mathrm{G}_{1}$ checkpoint. The mRNA expression levels of cyclin D1, as evaluated by RT-qPCR, were significantly reduced in chloroquine-treated SCC25 and CAL27 cells, in a dose-dependent manner, compared with the control cells $\left(\mathrm{P}<0.05\right.$; Fig. 2C). Cyclin D1 promotes the $\mathrm{G}_{1}-\mathrm{S}$ cell cycle transition; therefore, a reduction in its expression may result in growth inhibition.

Chloroquine inhibits autophagy. Chloroquine has been reported to inhibit autophagy by blocking fusion of the autophagosome and lysosome, thus resulting in a marked accumulation of autophagic vacuoles in numerous cell types, in which LC3 serves an important role. To determine whether the effects of chloroquine on OSCC cells were associated with autophagy, the levels of LC3 were detected. Chloroquine-treated SCC25 and CAL27 cells exhibited clumped LC3 immunoreactivity, indicating a chloroquine-induced accumulation of autophagic vacuoles, compared with relatively weak cytoplasmic LC3 expression in untreated cells. The subcellular distribution of LC3 immunoreactivity was monitored in SCC25 and CAL27 cells that were exposed to $30 \mu \mathrm{M}$ chloroquine for $24 \mathrm{~h}$ (Fig. 4A).

To confirm these immunocytochemical observations, the mRNA expression levels of LC3B were evaluated by RT-qPCR. The results demonstrated that the expression levels of LC3B were increased in a dose-dependent manner following treatment of SCC25 and CAL27 cells with chloroquine $(\mathrm{P}<0.05$; Fig. $4 \mathrm{~B})$. During autophagy, the cytosolic version (LC3-I) of LC3 is converted to the lipidized form (LC3-II), which is localized on the membrane of autophagosomes and autolysosomes (17). The expression levels of LC3-II were assessed by western blot analysis of whole cell lysates from cells collected at $24 \mathrm{~h}$ post-treatment with chloroquine $(0,10$ and $30 \mu \mathrm{M})$. LC3-II levels were increased after $10 \mu \mathrm{M}$ chloroquine treatment and demonstrated a dose-dependent increase. Representative data are presented for SCC25 and CAL27 cells (Fig. 4C).

Chloroquine inhibits OSCC tumor growth in vivo. To determine the antitumor activity of chloroquine in vivo, CAL27-bearing BALB/c nude mice were used as an OSCC xenograft nude murine model. The tumor volumes of female and male mice were 86.44 and $64.28 \mathrm{~mm}^{3}$ on the 7 th day, respectively; therefore, the mice were randomly assigned into two groups: The treatment group was treated with $50 \mathrm{mg} / \mathrm{kg} /$ day chloroquine, and the control group was treated with normal saline

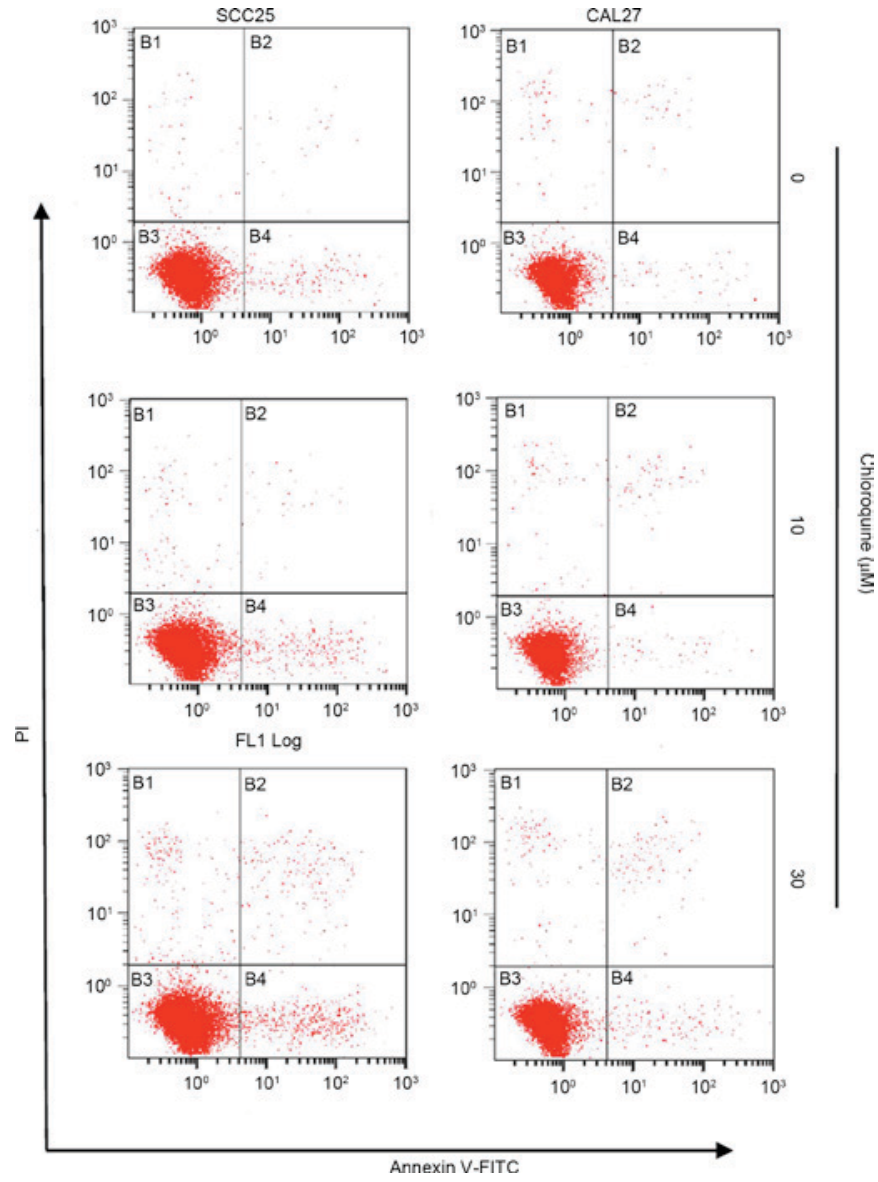

Figure 3. Chloroquine does not induce apoptosis of OSCC cells. SCC25 and CAL27 OSCC cells were incubated with chloroquine $(0,10$ and $30 \mu \mathrm{M})$ for $24 \mathrm{~h}(\mathrm{n}=3)$, labeled with Annexin V and PI, and analyzed by flow cytometry. The bottom right quadrant (Annexin $\mathrm{V}^{+} \mathrm{PI}^{-}$) represents early apoptotic cells, whereas the top right quadrant $\left(\right.$ Annexin $\mathrm{V}^{+} \mathrm{PI}^{+}$) represents late apoptotic cells. Chloroquine treatment increased the percentage of apoptotic cells in both OSCC cell lines tested; however, this difference was not statistically significant $(\mathrm{P}>0.05)$. OSCC, oral squamous cell carcinoma; FITC, fluorescein isothiocyanate; PI, propidium iodide.

solution. During the 24 days of treatment, all mice appeared to be healthy and there were no obvious signs or symptoms of drug toxicity. Consistent with the in vitro results of the present study, intraperitoneal administration of chloroquine reduced tumor growth in female and male CAL27-bearing BALB/c nude mice (Fig. 5A-C). In addition, the mean weights of the excised tumors were 25.68 and $38.28 \%$ lower in chloroquine-treated female and male mice compared with in control mice $(\mathrm{P}<0.05$; Fig. 5D).

\section{Discussion}

Chloroquine is an important antimalarial and antimycotic agent that is clinically safe and readily tolerated by humans. Previous studies have suggested that chloroquine may exert antitumor effects via cell cycle-, apoptosis-, proliferationand autophagy-associated mechanisms in several types of cancer cells (9-14). In addition, it has been demonstrated that chloroquine can overcome chemoresistance or increase radiosensitivity and eliminate cancer stem cells (18-21). However, the anticancer effects of chloroquine on OSCC remain to be elucidated; OSCC is the sixth most common 
A

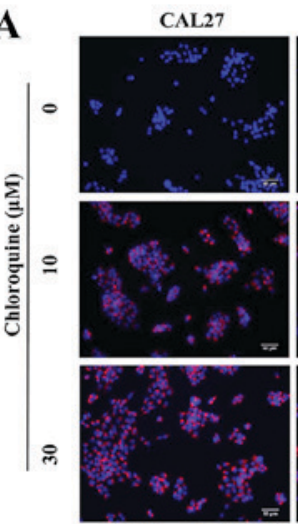

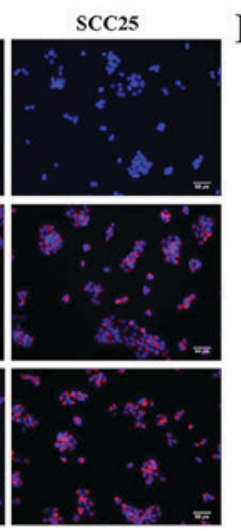
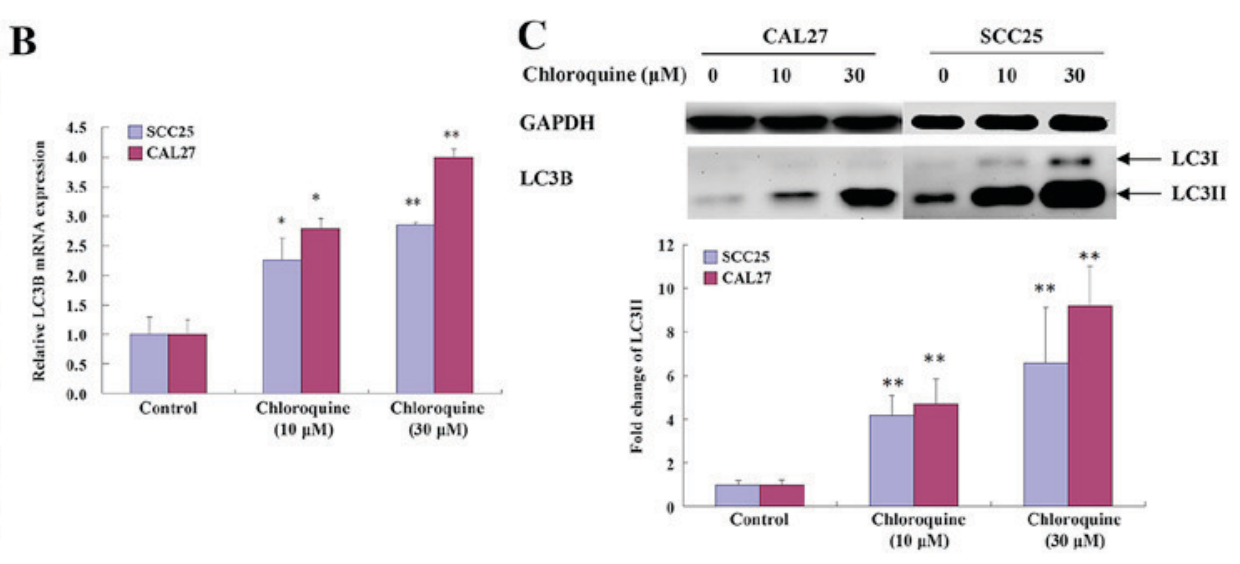

Figure 4. Chloroquine inhibits autophagy by inducing the accumulation of LC3B. (A) Intracellular localization of LC3B was determined by staining with fluorescent anti-LC3B antibodies; staining was analyzed by immunofluorescence microscopy. Control cells exhibited weak and diffuse cytoplasmic staining with LC3B-associated red fluorescence, whereas chloroquine-treated OSCC cells exhibited an evident punctuate red fluorescence pattern of LC3B, which is a typical feature of LC3B distribution within autophagosomes. (B) mRNA expression levels of LC3B were quantified in SCC25 and CAL27 cells by quantitative polymerase chain reaction. Cells treated with chloroquine exhibited a dose-dependent increase in LC3B. ${ }^{*} \mathrm{P}<0.05,{ }^{* *} \mathrm{P}<0.01$ vs. control cells. (C) Protein expression levels of LC3-II were determined by western blotting in SCC25 and CAL27 cells. Chloroquine treatment resulted in a dose-dependent increase in the accumulation of LC3-II. The expression levels of LC3-II are expressed as the density measured by ImageJ software, standardized to the density of GAPDH. OSCC, oral squamous cell carcinoma; LC3B, microtubule-associated proteins $1 \mathrm{~A} / 1 \mathrm{~B}$ light chain $3 \mathrm{~B} .{ }^{*} \mathrm{P}<0.05,{ }^{* * *} \mathrm{P}<0.01$ vs. control cells.

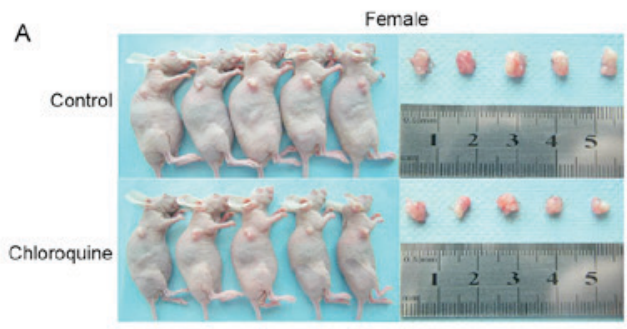

B

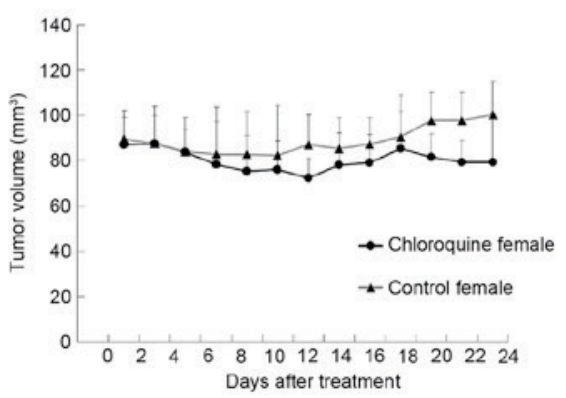

C

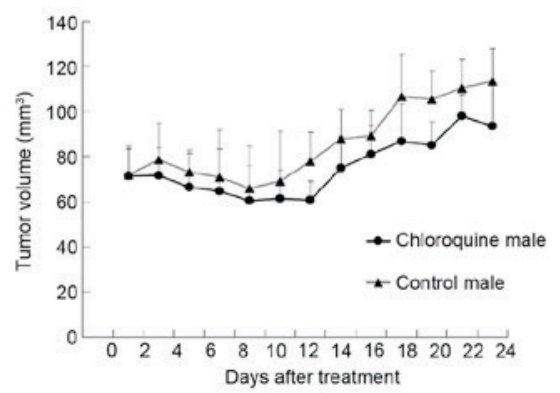

Male

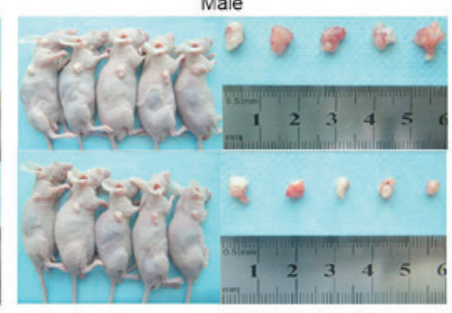

D

Figure 5. Chloroquine inhibits oral squamous cell carcinoma tumor growth in a CAL27 xenograft nude mouse model. (A) Images of the gross tumors from nude mice treated with chloroquine or normal saline. (B and C) Tumor growth curve represented by the average tumor volumes of CAL27 xenograft nude mice from the control and chloroquine-treated groups. Chloroquine treatment reduced the tumor growth rate in CAL27 xenograft nude mice. (D) Average weights of tumors excised from chloroquine-treated female and male mice were 25.68 and $38.28 \%$ lower than control mice, respectively. ${ }^{*} \mathrm{P}<0.05,{ }^{* *} \mathrm{P}<0.01 \mathrm{vs}$. control.

cancer worldwide, and patients with OSCC have high levels of morbidity and mortality. Due to poor prognosis, local recurrence and the difficulties associated with functional reconstruction, OSCC is considered a great therapeutic challenge. The present study aimed to investigate the effects of chloroquine on human OSCC cells and to determine the possible underlying mechanism. The results indicated that chloroquine can effectively inhibit the proliferation and colony-forming ability of OSCC cancer cells in vitro. Furthermore, the anticancer effects of chloroquine were associated with cell cycle arrest and autophagic inhibition in OSCC. Subsequently, an in vivo study was conducted to confirm the role of chloroquine as a potent therapeutic agent against OSCC.

Previous studies have reported that chloroquine exerts antitumor effects on several types of cancer; however, the underlying molecular target and mechanism remain to be elucidated (9-14). The present study investigated the effects of chloroquine on cell proliferation by MTT and clonogenic assays. The results demonstrated that both OSCC cell lines treated with chloroquine exhibited a reduction in proliferation in a dose- and time-dependent manner. Furthermore, a dose-dependent decrease in OSCC cell colony formation was induced following chloroquine treatment. A meta-analysis 
previously demonstrated that overexpression of cyclin D1 was significantly correlated with increased tumor size, lymphoid node metastasis, tumor differentiation, advancement of clinical stages, and adversely influenced overall survival of patients with OSCC (22-24). In addition, cyclin D1 polymorphism is associated with an increased susceptibility to OSCC (25). Therefore, cyclin D1 is considered a useful prognostic factor and therapeutic target. In the present study, chloroquine induced $\mathrm{G}_{0} / \mathrm{G}_{1}$ arrest in CAL27 and SCC25 cells $24 \mathrm{~h}$ post-treatment, and downregulated the expression of cyclin D1. Disturbed cell cycle progression may be a potential mechanism through which chloroquine inhibits OSCC cell growth.

Apoptosis is an important mechanism of antitumor drug-induced cell death, and the susceptibility of tumor cells to apoptosis is an important determinant of chemotherapeutic efficacy. Previous studies demonstrated that chloroquine is able to induce the apoptosis of various cell types, including human lung cancer cells, melanoma cells, colon cancer cells, breast cancer cells and hepatocellular carcinoma cells, the majority of which are adenocarcinomas (9-14). However, the present study detected only a slight increase in the percentage of apoptotic cells following treatment with $30 \mu \mathrm{M}$ chloroquine. This contradiction may be attributed to histological difference, which is one of the most important factors associated with the different patient responses to the same anticancer treatment. To the best of our knowledge, no previous studies have reported the chloroquine-induced apoptosis of squamous cell carcinoma cells; however, previous studies have demonstrated that the addition of chloroquine can promote the apoptosis of squamous cell carcinoma cells induced by cisplatin and the flavonoid luteolin (26-28). Therefore, future studies should aim to investigate the effects of chloroquine on various squamous cell carcinoma cell lines, using different monitoring methods.

It has previously been reported that targeting autophagy may be considered a novel anticancer therapeutic approach, and autophagy is a mediator of chemotherapy-induced cell death in cancer $(3,29)$. Both chloroquine and hydroxychloroquine are derivatives of a 4-aminoquinoline nucleus; hydroxychloroquine is considered the safer alternative, and both drugs can inhibit autophagy by blocking lysosome acidification and autophagosome degradation. Previous studies demonstrated that chloroquine and hydroxychloroquine act as novel antitumor drugs in various tumors by inhibiting autophagy; furthermore, chloroquine or hydroxychloroquine has been used alongside chemoradiotherapy in clinical trials for patients with refractory malignancies $(1,3,29)$. Autophagy is a frequent and early event during oral carcinogenesis, which may affect the malignant process of OSCC. Beclin-1, or autophagy-related 6, is an important mediator of autophagy, which has been reported to be significantly downregulated in OSCC tumor tissue, compared with normal tissue (30). Nomura et al demonstrated that overexpression and altered subcellular localization of autophagy-related 16-like 1 in oral premalignant lesions and primary OSCC was correlated with lymphovascular invasion and lymph-node metastasis (31). To investigate the mechanism underlying the inhibitory effects of chloroquine on proliferation, the present study examined whether chloroquine could suppress autophagy, using the LC3 conjugation system as an autophagosomal marker (17). The results indicated that chloroquine induced LC3 accumulation and increased LC3 expression at the mRNA and protein level, thus suggesting that chloroquine treatment may induce accumulation of damaged autolysosomes and suppress the progression of autophagy. This result is consistent with the findings of Geng et al, which reported that chloroquine may induce an accumulation of autophagic vacuoles in five glioma cell lines, indicating that chloroquine-induced cell death was associated with autophagy, but not with caspase-mediated apoptosis (13).

The present study investigated the in vivo antitumor effects of chloroquine against a CAL27-bearing BALB/c nude mouse model. As referred to in a previous in vivo study regarding the effects of chloroquine on colon cancer, $50 \mathrm{mg} / \mathrm{kg}$ chloroquine was used in the present study (10). The results of the in vivo study indicated that chloroquine effectively inhibited OSCC tumor growth, without being associated with signs or symptoms of drug toxicity. Notably, the present study is the first, to the best of our knowledge, to indicate that chloroquine may inhibit OSCC tumor growth in vitro and in vivo.

In conclusion, the results of the present study indicated that chloroquine is a potent compound that may inhibit OSCC cell proliferation and tumor growth, and warrants further study as a therapeutic agent against human OSCC.

\section{Acknowledgements}

The present study was supported by the Project of National Natural Sciences Foundation of China (grant no. 81272948) and the Guangdong Medical Scientific Research Fund (grant no. B2014446).

\section{References}

1. Kimura T, Takabatake Y, Takahashi A and Isaka Y: Chloroquine in cancer therapy: A double-edged sword of autophagy. Cancer Res 73: 3-7, 2013.

2. Al-Bari MA: Chloroquine analogues in drug discovery: New directions of uses, mechanisms of actions and toxic manifestations from malaria to multifarious diseases. J Antimicrob Chemother 70: 1608-1621, 2015.

3. Yang ZJ, Takabatake Y, Takahashi A and Isaka Y: The role of autophagy in cancer: Therapeutic implications. Mol Cancer Ther 10: 1533-1541, 2011.

4. Johnson NW, Jayasekara P and Amarasinghe AA: Squamous cell carcinoma and precursor lesions of the oral cavity: Epidemiology and aetiology. Periodontol 2000 57: 19-37, 2011.

5. Pignon JP, Bourhis J, Domenge $C$ and Designé L: Chemotherapy added to locoregional treatment for head and neck squamous-cell carcinoma: Three meta-analyses of updated individual data. MACH-NC collaborative group. Meta-analysis of chemotherapy on head and neck cancer. Lancet 355: 949-955, 2000.

6. Sturgis EM, Moore BA, Glisson BS, Kies MS, Shin DM and Byers RM: Neoadjuvant chemotherapy for squamous cell carcinoma of the oral tongue in young adults: A case series. Head Neck 27: 748-756, 2005.

7. Freier K, Engel M, Lindel K, Flechtenmacher C, Mühling J, Hassfeld $\mathrm{S}$ and Hofele C: Neoadjuvant concurrent radiochemotherapy followed by surgery in advanced oral squamous cell carcinoma (OSCC): A retrospective analysis of 207 patients. Oral Oncol 44: 116-123, 2008.

8. Wedemeyer I, Kreppel M, Scheer M, Zöller JE, Büttner R and Drebber U: Histopathological assessment of tumour regression, nodal stage and status of resection margins determines prognosis in patients with oral squamous cell carcinoma treated with neoadjuvant radiochemotherapy. Oral Dis 20: e81-e89, 2014. 
9. Jiang PD, Zhao YL, Shi W, Deng XQ, Xie G, Mao YQ, Li ZG, Zheng YZ, Yang SY and Wei YQ: Cell growth inhibition, G2/M cell cycle arrest and apoptosis induced by chloroquine in human breast cancer cell line Bcap-37. Cell Physiol Biochem 22: 431-440, 2008.

10. Zheng Y, Zhao YL, Deng X, Yang S, Mao Y, Li Z, Jiang P, Zhao $\mathrm{X}$ and Wei $\mathrm{Y}$ : Chloroquine inhibits colon cancer cell growth in vitro and tumor growth in vivo via induction of apoptosis. Cancer Invest 27: 286-292, 2009.

11. Hu T, Li P, Luo Z, Chen X, Zhang J, Wang C, Chen P and Dong Z: Chloroquine inhibits hepatocellular carcinoma cell growth in vitro and in vivo. Oncol Rep 35: 43-49, 2016.

12. Fan C, Wang W, Zhao B, Zhang S and Miao J: Chloroquine inhibits cell growth and induces cell death in A549 lung cancer cells. Bioorg Med Chem 14: 3218-3222, 2006.

13. Geng Y, Kohli L, Klocke BJ and Roth KA: Chloroquine-induced autophagic vacuole accumulation and cell death in glioma cells is p53 independent. Neuro Oncol 12: 473-481, 2010.

14. Lakhter AJ, Sahu RP, Sun Y, Kaufmann WK, Androphy EJ, Travers JB and Naidu SR: Chloroquine promotes apoptosis in melanoma cells by inhibiting BH3 domain-mediated PUMA degradation. J Invest Dermatol 133: 2247-2254, 2013.

15. Wang J, Jia L, Kuang Z, Wu T, Hong Y, Chen X, Leung WK, Xia J and Cheng B: The in vitro and in vivo antitumor effects of clotrimazole on oral squamous cell carcinoma. PLoS One 9: e98885, 2014.

16. Livak KJ and Schmittgen TD: Analysis of relative gene expression data using real-time quantitative PCR and the 2(-Delta Delta C(T)) Method. Methods 25: 402-408, 2001.

17. Tanida I, Ueno $\mathrm{T}$ and Kominami E: LC3 conjugation system in mammalian autophagy. Int J Biochem Cell Biol 36: 2503-2518, 2004.

18. Pascolo S: Time to use a dose of Chloroquine as an adjuvant to anti-cancer chemotherapies. Eur J Pharmacol 771: 139-144, 2016.

19. Lefort S, Joffre C, Kieffer Y, Givel AM, Bourachot B, Zago G, Bieche I, Dubois T, Meseure D, Vincent-Salomon A, et al: Inhibition of autophagy as a new means of improving chemotherapy efficiency in high-LC3B triple-negative breast cancers Autophagy 10: 2122-2142, 2014.

20. Liang DH, Choi DS, Ensor JE, Kaipparettu BA, Bass BL and Chang JC: The autophagy inhibitor chloroquine targets cancer stem cells in triple negative breast cancer by inducing mitochondrial damage and impairing DNA break repair. Cancer Lett 376: 249-258, 2016

21. Choi DS, Blanco E, Kim YS, Rodriguez AA, Zhao H, Huang TH, Chen CL, Jin G, Landis MD, Burey LA, et al: Chloroquine eliminates cancer stem cells through deregulation of Jak2 and DNMT1. Stem Cells 32: 2309-2323, 2014.
22. Angadi PV and Krishnapillai R: Cyclin D1 expression in oral squamous cell carcinoma and verrucous carcinoma: Correlation with histological differentiation. Oral Surg Oral Med Oral Pathol Oral Radiol Endod 103: e30-e35, 2007.

23. Zhong LP, Zhu DW, William WN Jr, Liu Y, Ma J, Yang CZ, Yang X, Wang LZ, Li J, Myers JN, et al: Elevated cyclin D1 expression is predictive for a benefit from TPF induction chemotherapy in oral squamous cell carcinoma patients with advanced nodal disease. Mol Cancer Ther 12: 1112-1121, 2013.

24. Zhao Y, Yu D, Li H, Nie P, Zhu Y, Liu S, Zhu M and Fang B: Cyclin D1 overexpression is associated with poor clinicopathological outcome and survival in oral squamous cell carcinoma in Asian populations: Insights from a meta-analysis. PLoS One 9: e93210, 2014

25. Myo K, Uzawa N, Miyamoto R, Sonoda I, Yuki Y and Amagasa T: Cyclin D1 gene numerical aberration is a predictive marker for occult cervical lymph node metastasis in TNM Stage I and II squamous cell carcinoma of the oral cavity. Cancer 104: 2709-2716, 2005

26. Zhao XG, Sun RJ, Yang XY, Liu DY, Lei DP, Jin T and Pan XL: Chloroquine-enhanced efficacy of cisplatin in the treatment of hypopharyngeal carcinoma in xenograft mice. PLoS One 10: e0126147, 2015.

27. Verschooten L, Barrette K, Van Kelst S, Rubio Romero N, Proby C, De Vos R, Agostinis P and Garmyn M: Autophagy inhibitor chloroquine enhanced the cell death inducing effect of the flavonoid luteolin in metastatic squamous cell carcinoma cells. PLoS One 7: e48264, 2012.

28. Quan HY, Quan HY, Zhou LJ, Li AD and Zhang ZB: Mechanism of chloroquine in promoting sensitivity of chemotherapeutics in oral squamous cell carcinoma CAL-27 cell line to cisplatin. Shanghai Kou Qiang Yi Xue 24: 30-36, 2015 (In Chinese).

29. Amaravadi RK, Lippincott-Schwartz J, Yin XM, Weiss WA, Takebe N, Timmer W, DiPaola RS, Lotze MT and White E: Principles and current strategies for targeting autophagy for cancer treatment. Clin Cancer Res 17: 654-666, 2011.

30. Kapoor V, Paliwal D, Baskar Singh S, Mohanti BK and Das SN: Deregulation of Beclin 1 in patients with tobacco-related oral squamous cell carcinoma. Biochem Biophys Res Commun 422: 764-769, 2012

31. Nomura H, Uzawa K, Yamano Y,Fushimi K, Ishigami T, Kouzu Y, Koike H, Siiba M, Bukawa H, Yokoe H, et al: Overexpression and altered subcellular localization of autophagy-related 16-like 1 in human oral squamous-cell carcinoma: Correlation with lymphovascular invasion and lymph-node metastasis. Hum Pathol 40 83-91, 2009. 